\title{
Hidden But Not Forgotten: The Trauma Experience Among Women with Severe Mental Illness and Our Role as Mental Health Professionals
}

\author{
Prabha S. Chandra
}

Received: 7 May 2019/Accepted: 7 May 2019/Published online: 17 May 2019

(C) Springer Nature India Private Limited 2019

Using a 'gender lens' while caring for women with severe mental illness (SMI) is important to be able to address some of their unique needs which may otherwise get neglected. Gender refers to socially constructed roles, behaviours and identities of being a woman or a man which play a major role in how people perceive themselves in relation to one another in society and the life experiences they go through.

Sex and gender interactions influence several aspects of lives among both men and women with SMI. Aspects like help seeking, trauma, early childhood adversities, nutrition and physical health are all influenced both by gender and sex. These may have specific impacts on the lived experience of mental illness and often on psychopathology as well. In addition, the role of the menstrual cycle and changes through a woman's lifespan may influence her mental illness in several ways. Not to mention the pharmacokinetics and pharmacodynamics of medications that result in differential side effects and effectiveness.

P. S. Chandra

Department of Psychiatry, National Institute of Mental Health and Neurosciences (NIMHANS), Bangalore, India

P. S. Chandra ( $\square)$

President Elect, International Association of Women's Mental Health, Bangalore, India

e-mail: chandra@nimhans.ac.in; prabhasch@gmail.com URL: http://www.perinatalpsynimhans.org/;

http://nimhans.ac.in/nimhans/psychiatry
Often as clinicians while we may pay emphasis to the more biological and medical aspects of sex, we tend to ignore some critical aspects related to gender when we care for women with severe mental illness. Women on the other hand may find it too hard to talk about these issues or may not think it is important and therefore not mention it. Often they may not have the words or language to express the distress associated with some of these experiences. On the other hand clinicians might be so overwhelmed by the mental illness and its consequences that they do not bring up these for discussion.

In recent years, the need to address trauma among persons with severe mental illness has gained importance. The recent definition by SAMHSA captures the gist of trauma.

'Individual trauma results from an event, series of events, or set of circumstances that is experienced by an individual as physically or emotionally harmful or life threatening and that has lasting adverse effects on the individual's functioning and mental, physical, social, emotional or spiritual well-being.'

Substance Abuse and Mental Health Services Administration [1].

It is now abundantly clear that women (and men) with SMI experience trauma probably more than those without any mental illness, that trauma has an important role in how it shapes the course and psychopathology of mental illness and that traumatic 
experiences may even influence life span in those with SMI $[2,3]$.

Traumatic experiences include those which occur in childhood such as sexual abuse, bullying, body shaming and those which occur in adulthood such as intimate partner violence or sexual coercion. Several research studies have found high rates of multiple traumatic experiences among persons with SMI, with women reporting specific forms of trauma such as sexual abuse in childhood. There are not too many studies that specifically report how many women with SMI disclose history of trauma on their own compared to that reported as part of research studies. However, a recent systematic review of international research found that only between 0 and $22 \%$ of service users report being asked about abuse experiences [4].

\section{How Does Trauma Manifest Among Persons with Mental Illness?}

Like others who have faced trauma, persons with severe mental illness may also experience the fight, flight and freeze reactions in the face of triggers of the traumatic memory. Rage reactions, severe anxiety and dissociation which may not be entirely explained by the psychosis alone may actually be a manifestation of the trauma reaction. Many women may not be willing to discuss trauma directly and this may manifest as somatic symptoms including pelvic pain or other gynaecological symptoms like vaginal discharge.

Dissociative symptoms which are linked closely to trauma have been largely ignored as a part of schizophrenia by the newer categorical classificatory systems. From theoretical conceptualisation to methods of treatment, the approach towards dissociation and schizophrenia has been to slot them as distinct disorders that seldom meet. Dissociative disorders or symptoms are treated with psychological therapies, while schizophrenia is treated with antipsychotic medication. Despite this nosological divide, studies have shown high rates of dissociation among persons with schizophrenia. Experienced clinicians would agree that dissociative experiences are commonly seen in schizophrenia and need to be considered as co occurring pathological experiences. One study among persons with schizophrenia spectrum disorders showed the following prevalence of dissociative phenomena-dissociative amnesia (34\%), depersonalization (48\%), derealization (22\%), identity confusion (46\%), and identity alteration (56\%) [5]. Trauma has been linked to dissociation among persons with schizophrenia and substantial rates of childhood adversity and specific associations with adult dissociation seen among patients with psychosis who reported sexual abuse or paternal dysfunction in childhood. One study found a higher prevalence of non auditory hallucinations and a dose-response relationship with absue-with higher number of adversities being associated with a report of more than one modality of non auditory hallucination [6].

If indeed, a significant majority of our patients with severe mental illness show signs related to traumatic experience, it is important that mental health professionals address these, in addition to treating psychotic symptoms.

\section{Trauma Informed Care}

Several studies among mental health professionals have assessed knowledge, comfort and skills about assessing sexual and partner violence. Clinicians report finding it too difficult to assess partner violence and sexual trauma when there is acute psychopathology or when situations such as poor psychosocial support add to the complexity. Added to this is a lack of time in busy outpatient and inpatient services which does not provide the optimal environment for sensitive questioning. Clinical training programmes should therefore focus on developing skills as well as provision of knowledge on both assessing and responding to trauma among women with SMI.

To this end the World Psychiatric Association has recently published a competency based curriculum on domestic and sexual violence [7]. Based on this a psychiatrist (and indeed all mental health professionals) should be able to describe physical, psychological and sexual intimate partner violence and its prevalence including among vulnerable groups such as women with SMI, be able to describe the physical and psychological health sequelae associated with IPV and SV, demonstrate competency in enquiring about violence in a supportive and safe way, demonstrate the principles of psychological first-aid ("LIVES"), be aware of and be able to use basic trauma focused psychological interventions for conditions such as 
PTSD or refer to appropriate resources, have knowledge of local laws and resources and how to refer those who have faced such violence and demonstrate medical record documentation of the discussion.

Mental health professionals need to start providing trauma informed care. This involves asking our patients- not what is wrong with you? But 'what has happened to you? [8]'.

Rather than being a specific service or set of rules, trauma-informed approaches are a process of systemic ways to create environments and relationships that promote safety and prevent retraumatisation. This includes being sensitive about issues such as seclusion and restraint that might trigger traumatic memories or being in a large psychiatric ward that might heighten feelings of threat.

It is important that patients with schizophreniaspectrum disorders are routinely asked about a broad range of possible adverse childhood experiences in order to provide appropriate interventions. Early trauma may be a stressor in the aetiopathophysiology of schizophrenia, particularly for cases with treatment refractory positive symptoms, and may guide future treatment development.

The finding that a substantial proportion of people with severe mental illness can present with symptoms related to trauma has been addressed by the treatment guidance for schizophrenia from the National Institute for Health and Care Excellence (NICE) [9]. In fact, the document even acknowledges psychosis and its treatment as being potentially traumatic (involuntary treatment as a traumatic event). The guidance calls for all patients to be routinely assessed for trauma and its consequences. There is limited research on the safety and feasibility of using trauma focused psychological interventions among persons with SMI. However, one systematic review provided support for the use and efficacy of these interventions.

The current mainstay of treatment of severe mental illness is antipsychotic medication. Psychological therapies find a limited role. But not surprisingly, patients find the human connection more important and the most healing part of a doctor patient relationship [10].

At a recent conference I heard Professor Elyn Saks, Professor of Law and adjunct Professor of Psychiatry from the University of California, speak about her journey through schizophrenia and her recovery in which psychotherapy played an important role. Later, I was reading her book 'The Centre Cannot Hold' in which she discusses how talk therapy and psychoanalysis in addition to medication helped her navigate some of the most difficult challenges of the illness and her relationships with others [11]. As clinicians we must remember that patients with severe mental illness also need working through trauma just like others and maybe even more.

The role of traumatic experiences in the lives of our patients needs to be addressed with empathy and understanding. Trauma does not differentiate by gender. But some traumas are more in women than in men and what we learn when working with women can then be a stepping stone towards handling trauma with sensitivity in both genders.

\section{References}

1. Substance Abuse and Mental Health Services Administration. SAMHSA's Concept of Trauma and Guidance for a Trauma-Informed Approach (HHS Publication No. 2014, (SMA) 14-4884). SAMHSA.

2. Nguyen T, Hauck YL, Pedruzzi RA, Frayne J, Rock D, Dragovic M. Sexual health and sexual trauma in women with severe mental illness: an exploratory survey of Western Australian community mental health services. Health Care Women Int. 2017;38(7):705-14.

3. Aas M, Elvsåshagen T, Westlye LT, Kaufmann T, Athanasiu L, Djurovic S, Melle I, Meer D, Martin-Ruiz C, Steen NE, Agartz I, Andreassen OA. Telomere length is associated with childhood trauma in patients with severe mental disorders. Transl Psychiatry. 2019;9(1):97. https:// doi.org/10.1038/s41398-019-0432-7.

4. Read J, Harper D, Tucker I, et al. Do adult mental health services identify child abuse and neglect? a systematic review. Int J Mental Health Nurs. 2017;27:7-19.

5. Renard SB, Huntjens RJ, Lysaker PH, Moskowitz A, Aleman A, Pijnenborg GH. Unique and overlapping symptoms in schizophrenia spectrum and dissociative disorders in relation to models of psychopathology: a systematic review. Schizophr Bull. 2017;43(1):108-21.

6. Longden E, House AO, Waterman MG. Associations between nonauditory hallucinations, dissociation, and childhood adversity in first-episode psychosis. J Trauma Dissociation. 2016;17(5):545-60.

7. Stewart D, Chandra PS. International competency-based curriculum for mental health providers on intimate partner violence and sexual violence against women. World Psychiatry. 2017;16(2):223-4.

8. Sweeney A, Filson B, Kennedy A, Collinson L, Gillard S. A paradigm shift: relationships in trauma-informed mental health services. BJPsych Adv. 2018;24(5):319-33. 
9. National Collaborating Centre for Mental Health. Psychosis and Schizophrenia in adults: treatment and management. NICE Clinical Guideline 178. NICE, 2014. http://www. nice.org.uk/guidance/cg178/evidence/cg178-psychosis-andschizophrenia-in-adults-full-guideline3. Accessed 18 May 2019.

10. Read J, Ross CA. Psychological trauma and psychosis: another reason why people diagnosed schizophrenic must be offered psychological therapies. J Am Acad Psychoanal Dyn Psychiatry: 2003, Vol. 31. The Schizophrenic person and the benefits of the psychotherapies-seeking a PORT in the Storm, pp. 247-268.

11. Saks ER. The centre cannot hold-my journey through madness. New York: Hachette Book Group; 2015.

Publisher's Note Springer Nature remains neutral with regard to jurisdictional claims in published maps and institutional affiliations. 